\title{
On the Performance of Future Full-Duplex Relay Selection Networks
}

\author{
(Invited Paper)
}

\author{
Mohammad Galal Khafagy ${ }^{\S}$, Mohamed-Slim Alouini $^{\S}$, and Sonia Aïssa* \\ $\S$ King Abdullah University of Science and Technology (KAUST), Thuwal, Makkah Province, Saudi Arabia \\ * Institut National de la Recherche Scientifique (INRS-EMT), University of Quebec, Montreal, QC, Canada \\ Email: \{mohammad.khafagy, slim.alouini\}@kaust.edu.sa, aissa@emt.inrs.ca
}

\begin{abstract}
In this work, we evaluate the performance of relay selection over Nakagami- $m$ fading channels in the presence of a cluster of full-duplex decode-and-forward relays with selfinterference. Specifically, we derive the exact cumulative distribution function of the end-to-end signal-to-interference-plus-noise ratio in the presence of a direct source-destination link. When a direct link exists, all dual-hop paths become mutually correlated due to the common direct-link interference. The presented exact results enable the evaluation of the outage performance and system throughput for fixed-rate transmission systems. The theoretical findings are verified by numerical simulations, where the severity of fading effect in the residual self-interference link is also discussed.
\end{abstract}

Keywords-Full-Duplex; Opportunistic Relay Selection; Outage Probability; Self-Interference.

\section{INTRODUCTION}

The International Telecommunication Union (ITU) has been seriously progressing since early 2012 to set the stage for future fifth generation $(5 \mathrm{G})$ mobile networks. Currently, the Radiocommunication Sector of ITU (ITU-R) is about to finalize its recommendation vision of the prospective $5 \mathrm{G}$ networks. Further, a new focus group has been very recently established by its Telecommunication Standardization Sector (ITU-T), which also expects to complete its first study by the end of 2015. Despite being still underway, experts from industry and academia have been discussing the set of features such future networks are expected to exhibit. This set includes reduced energy consumption, simultaneous huge data traffic support for large number of users, and lower latencies that translate into higher quality of user experience. Among others, fullduplex radio, where radio frontends simultaneously transmit and receive over the same spectrum bands, is envisioned as one of the promising technology enablers for $5 \mathrm{G}$ communications [1]. Such a full-duplex technology, also when adopted on top of cooperative communication settings, is anticipated to offer an enhanced spectral and energy efficiency to future mobile networks. In this paper, we focus our attention on scenarios where full-duplex cooperation is utilized.

Recently, full-duplex communication has experienced major practical developments [2]-[5]. These developments motivated further research in cooperative contexts where simultaneous listening/forwarding was allowed by full-duplex relaying
$(F D R)$, thereby eliminating the known rate loss of half-duplex relays. However, due to simultaneous transmission/reception at the relay, self-interference is introduced which cannot be perfectly eliminated even with the use of state-of-theart isolation and cancellation techniques. This residual selfinterference (RSI) can seriously affect the performance of full-duplex systems if not adequately treated. In this work, we focus on studying the end-to-end outage performance and system throughput of decode-and-forward (DF) FDR when such a residual level of self-interference persists. The work on amplify-and-forward relaying or other performance metrics is beyond the scope of the current work, and the interested reader is referred to [6], [7] and the references therein.

The outage performance of DF-FDR has been recently studied for different relaying schemes and channel fading models. Under Rayleigh fading, exact outage probability expressions have been derived in [8] with the direct sourcedestination link treated as interference. Such a relaying scheme, where the information is only distilled from the multihop path, will be referred to as the multi-hop DF (MHDF) scheme in the sequel. Research efforts have been devoted to investigate the performance under other relaying schemes where the direct link can be also exploited; using block Markov superposition coding (BMSC) [9] and selective relaying [10]. Under the more general Nakagami- $m$ fading model, the outage performance has been also studied for MHDF [11], BMSC [12], and selective relaying [13].

All the above-mentioned work considered settings where only a single relay node is available for cooperation. More recently, the potential gains of relay selection have been independently investigated in [14], [15] and in [16], also within an underlay cognitive radio setting where a primary system imposes interference limitations that should be maintained. Specifically, the end-to-end signal-to-interference-plus-noise ratio (SINR) distribution has been derived in closed-form assuming no direct link exists under Rayleigh [14], [16] and Nakagami- $m$ [15] fading. However, the performance analysis of the system becomes more challenging in the presence of a direct link. This is due to the fact that the multi-hop paths through all candidate relays become correlated due to the common interfering direct link. Also, in underlay scenarios, the superimposed interference signals at the primary receiver 


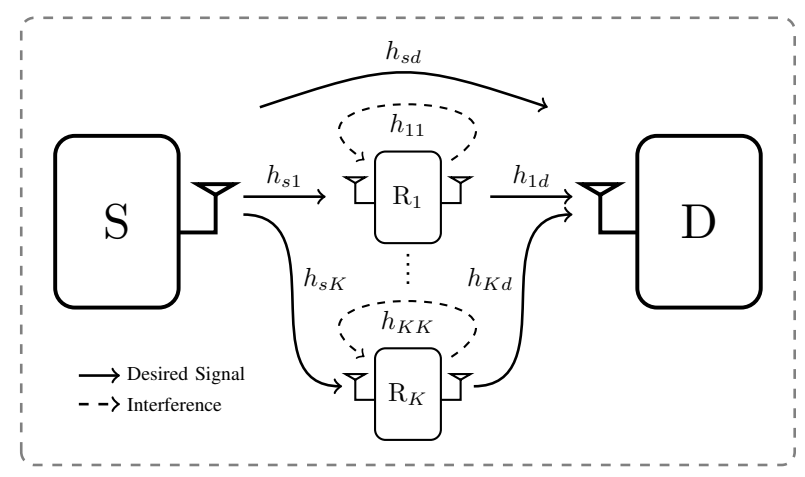

Fig. 1. A full-duplex cooperative setting with opportunistic relay selection.

from the secondary source and each of the relays become also correlated due to the common source interference. This latter problem has been investigated in [16] in Rayleighfading environments where exact closed-form expressions were derived and validated.

In this work, we evaluate the performance of opportunistic full-duplex relay selection with RSI and an interfering direct link, i.e., the MHDF relaying scheme is adopted by each cooperating relay. For such a relaying scheme, we obtain an exact closed-form expression for the end-to-end SINR cumulative distribution function (CDF) which readily yields the outage probability and system throughput for fixed-rate transmission systems. Also, we derive the exact SNR CDF for a hybrid scheme that exploits the direct link as an additional diversity branch. Finally, and via numerical simulations, we validate our exact theoretical results, and assess the effect of the severity of fading on the residual self-interference link .

\section{SySTEM MODEL}

We consider the communication setting depicted in Fig. 1 , where a source $(\mathrm{S})$ intends to communicate with a distant destination (D). The direct $\mathrm{S}-\mathrm{D}$ link is assumed of a relatively weak gain due to distance and shadowing effects. Accordingly, a full-duplex relay is utilized to assist the end-toend communication. FDR can offer higher spectral efficiency when compared to its half-duplex counterpart. However, FDR in practice suffers from RSI which imposes an additional communication challenge. In order to boost the system performance and alleviate the aforementioned interference effect, the source may select one relay out of a cluster of $K$ fullduplex relays, $\mathcal{R}=\left\{\mathrm{R}_{1}, \mathrm{R}_{2}, \cdots, \mathrm{R}_{K}\right\}$.

\section{A. Channel Model}

The fading coefficient of the $i-j$ link is denoted by $h_{i j}$, for $i \in\{s, 1,2, \cdots, K\}$ and $j \in\{1,2, \cdots, K, d\}$, where $s$ and $d$ refer to the source and destination nodes (Fig. 1) respectively, while $k \in \mathcal{K}$ denotes the relay index, with $\mathcal{K}=\{1,2, \cdots, K\}$ being the set of all possible relay indices. Moreover, the $i-j$ link gain is denoted by $g_{i j}=\left|h_{i j}\right|^{2}$. All channels are assumed to follow a block fading model, where $h_{i j}$ remains constant over one block, and varies independently from one block to another following a Nakagami-m fading model with shape parameter $m_{i j}$ and average power $\mathbb{E}\left\{\left|h_{i j}\right|^{2}\right\}=\pi_{i j}$. Accordingly, the channel gain, $\left|h_{i j}\right|^{2}$, is a Gamma random variable
$(\mathrm{RV})$ with shape parameter $m_{i j}$ and scale parameter $\theta_{i j}=\frac{\pi_{i j}}{m_{i j}}$, for which we use the shorthand notation $\mathcal{G}\left(m_{i j}, \theta_{i j}\right)$. For a Gamma RV $X \sim \mathcal{G}(m, \theta)$, its probability density function (PDF) and $\mathrm{CDF}$ are respectively given by

$f_{X}(x ; m, \theta)=\frac{x^{m-1} e^{-\frac{x}{\theta}}}{\Gamma(m) \theta^{m}}$ and $F_{X}(x ; m, \theta)=\frac{\gamma\left(m, \frac{x}{\theta}\right)}{\Gamma(m)}$,

where $\gamma(a, b)=\int_{0}^{b} t^{a-1} e^{-t} d t$ denotes the lower incomplete Gamma function and $\Gamma(a)$ denotes the Gamma function [17]. All channel fading gains are assumed to be mutually independent. The relays operate in a full-duplex mode where simultaneous listening/forwarding is allowed with an introduced level of loopback interference. The link gain $h_{k k}$ is assumed to represent the RSI after undergoing all possible isolation and cancellation techniques, as for instance [6] and the references therein. The source and the $k^{\text {th }}$ relay powers are denoted by $P_{s}$ and $P_{k}$, respectively. Also, $n_{k}$ and $n_{d}$ denote the complex additive white Gaussian noise components at the $k^{\text {th }}$ relay and the destination, with variance $\sigma_{k}^{2}$ and $\sigma_{d}^{2}$, respectively.

As commonly assumed in the literature, for instance in [18], we assume the relays are clustered somewhere between the source and the destination. Hence, the distances among the relays are much shorter than those between the relays and the source/destination. Thus, it is reasonable to assume the following symmetric scenario where all source-relay links have an average gain of $\mathbb{E}\left\{g_{s k}\right\}=\pi_{s r}$, while all relay-destination links have an average gain of $\mathbb{E}\left\{g_{k d}\right\}=\pi_{r d}, \forall k \in \mathcal{K}$. Also, we assume that all relays have their loopback interference links with the same average gain, i.e., $\mathbb{E}\left\{g_{k k}\right\}=\pi_{r r}$. Further, it is assumed that $m_{s k}=m_{s r}, m_{k d}=m_{r d}$ and $m_{k k}=m_{r r}$ for all $k \in \mathcal{K}$. Although the analysis of asymmetric scenarios remains possible, the previous assumptions allow for simpler final expressions, and yet maintain the same diversity order of the system. Therefore, it follows that $\theta_{s k}=\theta_{s r}, \theta_{k d}=$ $\theta_{r d}$ and $\theta_{k k}=\theta_{r r}$ for all $k \in \mathcal{K}$. Finally, we assume that $\sigma_{k}^{2}=\sigma_{d}^{2}=1$, while all transmit powers are absorbed into the channel coefficients for ease of notation.

\section{B. Signal Model}

When the $k^{\text {th }}$ relay is selected, the received signals at the $k^{\text {th }}$ relay and destination at time $t$ are given, respectively, by

$$
\begin{aligned}
& y_{k}[t]=h_{s k} x_{s}[t]+h_{k k} x_{k}[t]+n_{k}[t], \\
& y_{d}[t]=h_{k d} x_{k}[t]+h_{s d} x_{s}[t]+n_{d}[t],
\end{aligned}
$$

where $x_{s}[t]$ and $x_{k}[t]$ are the source's transmit signal and the $k^{\text {th }}$ relay's forwarded signal at time $t$, respectively. When the $k^{\text {th }}$ relay successfully decodes, $x_{k}[t]=x_{s}[t-D]$ where $D$ is the relay transmission delay relative to that of the source in symbol times. Due to the source and relay asynchronous transmissions, the signal transmitted by the relay (source) is considered as an additional noise term at the relay (destination) as commonly treated in the related literature [6]. Accordingly, the received SINRs at the $k^{\text {th }}$ relay and at the destination are given respectively by

$$
\gamma_{s k}=\frac{g_{s k}}{g_{k k}+1} \quad \text { and } \gamma_{k d}=\frac{g_{k d}}{g_{s d}+1}
$$

In the following section, we analyze the performance of opportunistic full-duplex relay selection (FDRS) in the 
absence/presence of a direct S - D link.

\section{Performance Analysis of Opportunistic FDRS}

In this section, we derive the end-to-end SINR CDF for opportunistic FDRS with/without a direct $\mathrm{S}-\mathrm{D}$ link. Specifically, when $K$ decode-and-forward full-duplex relays are available, and in the absence of a direct link, the end-toend SINR is given by

$$
\gamma=\max _{k \in \mathcal{K}}\left\{\gamma_{k}\right\}
$$

where

$$
\gamma_{k}=\min \left\{\gamma_{s k}, \gamma_{k d}\right\} \text {. }
$$

The expression in (5) also applies to the scenario where the direct link exists, yet it is treated as mere interference. Alternatively, if direct transmission is taken into account as a possible diversity branch, the end-to-end SINR is given by

$$
\gamma=\max \left\{\max _{k \in \mathcal{K}}\left\{\gamma_{k}\right\}, g_{s d}\right\} .
$$

In what follows, we derive the exact end-to-end SINR CDF for three scenarios; 1) no direct link (NDL), 2) interfering direct link (IDL) and 3) hybrid multi-hop/direct transmission (MH/DT).

\section{A. Important Probability Distributions}

We first recall the following theorem from [11] for the CDF of a $\mathrm{RV} Z=\frac{X_{1}}{X_{2}+1}$, where $X_{1}$ and $X_{2}$ are independent and non identically distributed (i.n.i.d.) Gamma RVs.

Theorem 1 (CDF of $\left.Z=\frac{X_{1}}{X_{2}+1}\right)$ : The CDF of $Z=\frac{X_{1}}{X_{2}+1}$, where $X_{i} \sim \mathcal{G}\left(m_{i}, \theta_{i}\right)$, for $i \in\{1,2\}$, are i.n.i.d. RVs, for general real-valued $m_{1} \geq \frac{1}{2}$ and integer-valued $m_{2} \geq 1$, is given by [11], [12]:

$$
F_{Z}(z ; \boldsymbol{p})=\frac{\gamma\left(m_{1}, \frac{z}{\theta_{1}}\right)}{\Gamma\left(m_{1}\right)}+B \sum_{k=0}^{m_{2}-1} \frac{c^{-d}}{\theta_{2}{ }^{k}} W_{a, b}[c],
$$

where $\boldsymbol{p}=\left(m_{1}, \theta_{1}, m_{2}, \theta_{2}\right)$ is a vector of distribution parameters, $W_{a, b}[c]$ is the Whittaker function [17, Eq. 13.1.33], $a=\frac{m_{1}-k-1}{2}, b=\frac{-m_{1}-k}{2}, c=\frac{z}{\theta_{1}}+\frac{1}{\theta_{2}}, d=\frac{m_{1}+k+1}{2}$ and

$$
B=\frac{\exp \left(-\frac{1}{2}\left(\frac{z}{\theta_{1}}-\frac{1}{\theta_{2}}\right)\right)}{\Gamma\left(m_{1}\right)}\left(\frac{z}{\theta_{1}}\right)^{m_{1}} .
$$

The aforementioned distribution is important in analyzing systems involving RVs on the form in (4). In particular, despite being of a non-elementary form, such a distribution gives a general expression for full-duplex scenarios where the RSI channel still exhibits line-of-sight effects, i.e., scenarios where more emphasis is given to the employment of active cancellation techniques than passive cancellation/isolation [3]. However, depending on the communication setup, it is shown that passive cancellation in recent implementations can provide up to $65 \mathrm{~dB}$ cancellation, which eliminates the line-of-sight effects and renders Rayleigh fading a quite reasonable model for the residual channel [4], [5]. For such scenarios, the following simpler distribution is better used.
Corollary 1 (Special case when $m_{2}=1$ ): When $X_{2}$ is an exponential RV, i.e., $m_{2}=1$, the $\mathrm{CDF}$ of $Z=\frac{X_{1}}{X_{2}+1}$ is given, for $\boldsymbol{v}=\left(m_{1}, \theta_{1}, 1, \pi_{2}\right)$, by

$$
F_{Z}(z ; \boldsymbol{v})=\frac{\gamma\left(m_{1}, \frac{z}{\theta_{1}}\right)}{\Gamma\left(m_{1}\right)}+\frac{e^{\frac{1}{\pi_{2}}}}{\left(\frac{\theta_{1}}{z \pi_{2}}+1\right)^{m_{1}}} \frac{\Gamma\left(m_{1}, \frac{1}{\pi_{2}}+\frac{z}{\theta_{1}}\right)}{\Gamma\left(m_{1}\right)},
$$

where $\Gamma(a, b)=\int_{b}^{\infty} t^{a-1} e^{-t} d t$ denotes the upper incomplete Gamma function [17].

Proof: By conditioning/deconditioning on $X_{1}$, we have

$$
\begin{aligned}
F_{Z}(z ; \boldsymbol{v}) & =\int_{0}^{\infty} \mathbb{P}\left\{X_{2} \geq \frac{x_{1}-z}{z} \mid X_{1}=x_{1}\right\} \frac{x_{1}^{m_{1}-1} e^{-\frac{x_{1}}{\theta_{1}}}}{\Gamma\left(m_{1}\right) \theta_{1}^{m_{1}}} \mathrm{~d} x_{1} \\
& =\frac{\gamma\left(m_{1}, \frac{z}{\theta_{1}}\right)}{\Gamma\left(m_{1}\right)}+e^{\frac{1}{\pi_{2}}} \int_{z}^{\infty} \frac{x_{1}^{m_{1}-1} e^{-x_{1}\left(\frac{1}{z \pi_{2}}+\frac{1}{\theta_{1}}\right)}}{\Gamma\left(m_{1}\right) \theta_{1}^{m_{1}}} \mathrm{~d} x_{1},
\end{aligned}
$$

which yields (10) using [19, Eq. 3.381-3]. This can be verified to be a special case of (8) when $m_{2}=1$ using the relation between Whittaker's and Tricomi's confluent hypergeometric functions in [17, Eq. 13.1.33] and the special case of Tricomi's function in [17, Eq. 13.6.28].

\section{B. CDF of Link SINRs}

1) First hop: According to the channel model explained in Section II-A, the CDF of the SINR pertaining to the first hop of the $k^{\text {th }}$ path follows from Theorem 1 and Corollary 1 for the cases of Nakagami- $m$ and Rayleigh fading RSI channel, respectively, as

$$
F_{\gamma_{s k}}(\alpha)=F_{Z}\left(\alpha ; \boldsymbol{p}_{1}\right) \quad \text { and } \quad F_{\gamma_{s k}}(\alpha)=F_{Z}\left(\alpha ; \boldsymbol{v}_{1}\right) \text {, }
$$

where $\boldsymbol{p}_{1}=\left(m_{s r}, \theta_{s r}, m_{r r}, \theta_{r r}\right)$ and $\boldsymbol{v}_{1}=\left(m_{s r}, \theta_{s r}, 1, \pi_{r r}\right)$.

2) Second hop: Since the direct link gain, $g_{s d}$, is a common $\mathrm{RV}$ among all the multi-hop paths, all the second-hop gains are clearly correlated. However, they are conditionally independent given $g_{s d}=\beta$. Thus, in the presence of a direct link, we are only interested in the conditional distributions of the second-hop gains given $g_{s d}=\beta$, which follow $\gamma_{k d} \mid g_{s d} \sim$ $\mathcal{G}\left(m_{r d}, \frac{\theta_{r d}}{\beta+1}\right), \forall k \in \mathcal{K}$. On the other hand, when no direct link exists, it is clear that $\gamma_{k d} \mid g_{s d} \sim \mathcal{G}\left(m_{r d}, \theta_{r d}\right)$.

3) The $k^{\text {th }}$ multi-hop path: According to (6) and with the abovementioned distributions of the hop SINRs, the conditional CDF of the SINR over the $k^{t h}$ path given the direct link gain $g_{s d}=\beta$ is given by

$$
F_{\gamma_{k} \mid g_{s d}}(\alpha \mid \beta)=1-\overline{F_{Z}}\left(\alpha ; \boldsymbol{p}_{1}\right) \overline{F_{X}}\left(\alpha ; m_{r d}, \frac{\theta_{r d}}{\beta+1}\right),
$$

where $\bar{F}(\cdot)=1-F(\cdot)$ denotes the complementary CDF. It is clear that in the absence of a direct link, the CDF of the $k^{t h}$ path SINR is given by

$$
F_{\gamma_{k}}(\alpha)=1-\overline{F_{Z}}\left(\alpha ; \boldsymbol{p}_{1}\right) \overline{F_{X}}\left(\alpha ; m_{r d}, \theta_{r d}\right) .
$$

\section{End-to-end SINR CDF}

1) No direct link: In the absence of a direct $\mathrm{S}-\mathrm{D}$ link, the end-to-end SINR CDF has the following simple form:

$F_{\gamma}^{\mathrm{NDL}}(\alpha)=F_{\gamma_{k}}(\alpha)^{K}=\left(1-\overline{F_{Z}}\left(\alpha ; \boldsymbol{p}_{1}\right) \overline{F_{X}}\left(\alpha ; m_{r d}, \theta_{r d}\right)\right)^{K}$, 


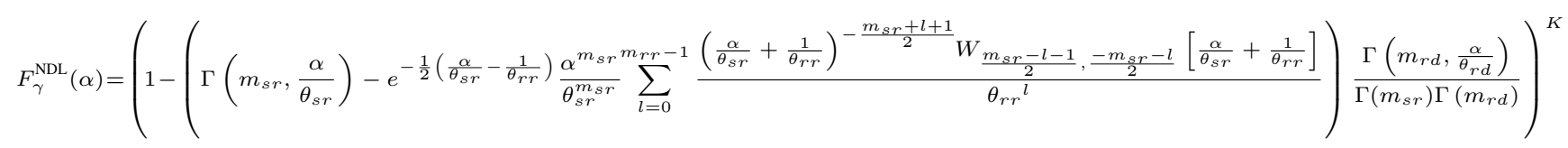

$$
F_{\gamma}^{\mathrm{NDL}}(\alpha)=\left(1-\left(\Gamma\left(m_{s r}, \frac{\alpha}{\theta_{s r}}\right)-\frac{e^{\frac{1}{\pi_{r r}}}\left(\alpha \pi_{r r}\right)^{m_{s r}} \Gamma\left(m_{s r}, \frac{\theta_{s r}+\alpha \pi_{r r}}{\theta_{s r \pi} \pi_{r r}}\right)}{\left(\theta_{s r}+\alpha \pi_{r r}\right)^{m_{s r}}}\right) \frac{\Gamma\left(m_{r d}, \frac{\alpha}{\theta_{r d}}\right)}{\Gamma\left(m_{s r}\right) \Gamma\left(m_{r d}\right)}\right)^{K}
$$

which is explicitly given in (16) on the top of the next page for real-valued $m_{s r}$ and $m_{r d}$, and integer-valued $m_{r r}$. For $m_{r r}=$ 1 , we get the simpler form in (17) via Corollary 1.

2) Interfering direct link: In this scenario, the end-to-end SINR CDF is given by

$$
\begin{aligned}
F_{\gamma}^{\mathrm{IDL}}(\alpha) & =\int_{0}^{\infty} F_{\gamma_{k} \mid g_{s d}}(\alpha \mid \beta)^{K} f_{x}\left(\beta ; m_{s d}, \theta_{s d}\right) \mathrm{d} \beta \\
& =\sum_{k=0}^{K} C(\alpha, k) \underbrace{\int_{0}^{\infty} \frac{\Gamma\left(m_{r d}, \frac{\alpha(\beta+1)}{\theta_{r d}}\right)^{k}}{\Gamma\left(m_{r d}\right)^{k}} \beta^{m_{s d}-1} e^{-\frac{\beta}{\theta_{s d}}} \mathrm{~d} \beta}_{\mathcal{I}_{1}},
\end{aligned}
$$

with

$$
C(\alpha, k)=\left(\begin{array}{c}
K \\
k
\end{array}\right) \frac{\left(-\overline{F_{Z}}\left(\alpha ; \boldsymbol{p}_{1}\right)\right)^{k}}{\Gamma\left(m_{s d}\right) \theta_{s d} m_{s d}},
$$

where the binomial expansion is exploited in the last step. We are now interested in solving the integral $\mathcal{I}_{1}$. Using the finite series expansion of the upper incomplete Gamma function in [19, Eq. 8.352-2], for integer values of $m_{r d}$, we get

$$
\begin{aligned}
\mathcal{I}_{1} & =\int_{0}^{\infty}\left(e^{-\frac{\alpha(\beta+1)}{\theta_{r d}}} \sum_{n=1}^{m_{r d}} \frac{\left(\frac{\alpha(\beta+1)}{\theta_{r d}}\right)^{n-1}}{\Gamma(n)}\right)^{k} \beta^{m_{s d}-1} e^{-\frac{\beta}{\theta_{s d}}} \mathrm{~d} \beta \\
= & e^{-\frac{\alpha k}{\theta_{r d}}} \sum_{\sum_{n=1}^{m_{r d} k_{n}=k}} C_{\left\{k_{n}\right\}} \mathcal{I}_{2},
\end{aligned}
$$

where

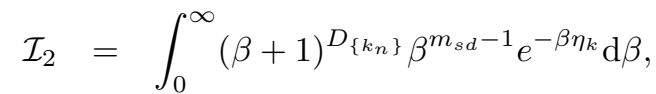

$$
\begin{aligned}
& \eta_{k}=\left(\frac{1}{\theta_{s d}}+\frac{\alpha k}{\theta_{r d}}\right) \text {, } \\
& C_{\left\{k_{n}\right\}}=\frac{\Gamma(k+1)\left(\frac{\alpha}{\theta_{r d}}\right)^{\sum_{n=1}^{m_{r d}} k_{n}(n-1)}}{\prod_{n=1}^{m_{r d}} \Gamma\left(k_{n}+1\right) \Gamma(n)^{k_{n}}}, \\
& D_{\left\{k_{n}\right\}}=\sum_{n=1}^{m_{r d}} k_{n}(n-1) \text {, }
\end{aligned}
$$

with (19) obtained via the multinomial theorem [17, Section 24.1.2]. By substitution of variables, $y=\beta+1$, the integral $\mathcal{I}_{2}$ in (20) is on the form of the Riemann-Liouville integral in
[19, Eq. 3.383-4]. Hence,

$$
\begin{aligned}
& \mathcal{I}_{2}=e^{\eta_{k}} \int_{1}^{\infty} y^{D_{\left\{k_{n}\right\}}}(y-1)^{m_{s d}-1} e^{-y \eta_{k}} \mathrm{~d} y \\
& =e^{\frac{\eta_{k}}{2}} \eta_{k}^{-\frac{m_{s d}+D_{\left\{k_{n}\right\}}+1}{2}} \Gamma\left(m_{s d}\right) \\
& \times W_{\frac{D_{\left\{k_{n}\right\}}-m_{s d}+1}{2}},-\frac{m_{s d}+D_{\left\{k_{n}\right\}}}{2}\left[\eta_{k}\right] .
\end{aligned}
$$

Accordingly, $F_{\gamma}^{\mathrm{IDL}}(\alpha)$ is given by (25) shown on the top of next page for integer-valued $m_{r r}$ and $m_{r d}$, and real-valued $m_{s r}$ and $m_{s d}$. In the special case when the $\mathrm{R}-\mathrm{D}$ link is Rayleigh-fading, i.e., $m_{r d}=1$, much simpler expressions can be obtained which also avoids the use of the multinomial theorem. Specifically, the integral $\mathcal{I}_{1}$ reduces to

$\mathcal{I}_{1}=e^{-\frac{\alpha k}{\theta_{r d}}} \int_{0}^{\infty} \beta^{m_{s d}-1} e^{-\beta \eta_{k}} \mathrm{~d} \beta=e^{-\frac{\alpha k}{\theta_{r d}}} \Gamma\left(m_{s d}\right) \eta_{k}^{-m_{s d}}$,

using [19, Eq. 3.351-3].

3) Hybrid multi-hop/direct transmission: When the direct $\mathrm{S}-\mathrm{D}$ link is leveraged as an additional diversity path, $g_{s d}=\beta$ is bounded above by $\alpha$. Hence, the CDF expression is similar to that of the IDL case, however with the upper integration limit changed to $\alpha$. That is,

$$
F_{\gamma}^{\mathrm{MH} / \mathrm{DT}}(\alpha)=\int_{0}^{\alpha} F_{\gamma_{k} \mid g_{s d}}(\alpha \mid \beta)^{K} f_{x}\left(\beta ; m_{s d}, \theta_{s d}\right) \mathrm{d} \beta .
$$

The evaluation of such an integral follows the same steps, except that the integral $\mathcal{I}_{2}$ is changed to

$$
\begin{aligned}
& \tilde{\mathcal{I}}_{2}=\int_{0}^{\alpha}(\beta+1)^{D_{\left\{k_{n}\right\}}} \beta^{m_{s d}-1} e^{-\beta \eta_{k}} \mathrm{~d} \beta \\
& =\sum_{r=0}^{D_{\left\{k_{n}\right\}}}\left(\begin{array}{c}
D_{\left\{k_{n}\right\}} \\
r
\end{array}\right) \int_{0}^{\alpha} \beta^{r+m_{s d}-1} e^{-\beta \eta_{k}} \mathrm{~d} \beta \\
& =\sum_{r=0}^{D_{\left\{k_{n}\right\}}}\left(\begin{array}{c}
D_{\left\{k_{n}\right\}} \\
r
\end{array}\right) \eta_{k}^{-\left(r+m_{s d}\right)} \gamma\left(r+m_{s d}, \alpha \eta_{k}\right) \text {, }
\end{aligned}
$$

for integer-valued $m_{s d}$ due to the use of the binomial theorem, with the last integral evaluated using [19, Eq. 3.381-1]. Hence, $F_{\gamma}^{\mathrm{MH} / \mathrm{DT}}(\alpha)$ is finally given by (31) for real-valued $m_{s r}$ and integer-valued $m_{r r}, m_{r d}$ and $m_{s d}$. Also, simpler expressions can be obtained in the special case when $m_{r d}=1$ since the integral $\tilde{\mathcal{I}}_{1}\left(\mathcal{I}_{1}\right.$ with the upper integral changed to $\alpha$ ) reduces to

$$
\begin{aligned}
\tilde{\mathcal{I}}_{1} & =e^{-\frac{\alpha k}{\theta_{r d}}} \int_{0}^{\alpha} \beta^{m_{s d}-1} e^{-\beta \eta_{k}} \mathrm{~d} \beta \\
& =e^{-\frac{\alpha k}{\theta_{r d}}} \gamma\left(m_{s d}, \alpha \eta_{k}\right) \eta_{k}^{-m_{s d}},
\end{aligned}
$$

using [19, Eq. 3.351-1]. 


$$
\begin{aligned}
& F_{\gamma}^{\operatorname{IDL}}(\alpha)=\sum_{k=0}^{K}\left(\begin{array}{l}
K \\
k
\end{array}\right) \frac{e^{\left(\frac{1}{2 \theta_{s d}}-\frac{\alpha k}{2 \theta_{r d}}\right)}\left(e^{-\frac{1}{2}\left(\frac{\alpha}{\theta_{s r}}-\frac{1}{\theta_{r r}}\right)} \frac{\alpha m_{s r}}{\theta_{s r}^{m} m_{r r}} \sum_{l=0}^{m_{r r}-1} \frac{\left.\left(\frac{\alpha}{\theta_{s r}}+\frac{1}{\theta_{r r}}\right)^{-\frac{m_{s r}+l+1}{2}} W_{\frac{m_{s r}-l-1}{2},-\frac{m_{s r}-l}{2}\left[\frac{\alpha}{\theta_{s r}}+\frac{1}{\theta_{r r}}\right]}-\Gamma\left(m_{s r}, \frac{\alpha}{\theta_{s r}}\right)\right)^{k}}{\theta_{r r}^{r}}\right.}{\theta_{s d} m_{s d} \Gamma\left(m_{s r}\right)^{k}}
\end{aligned}
$$

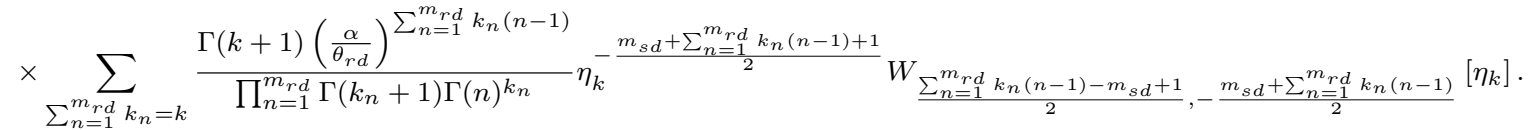

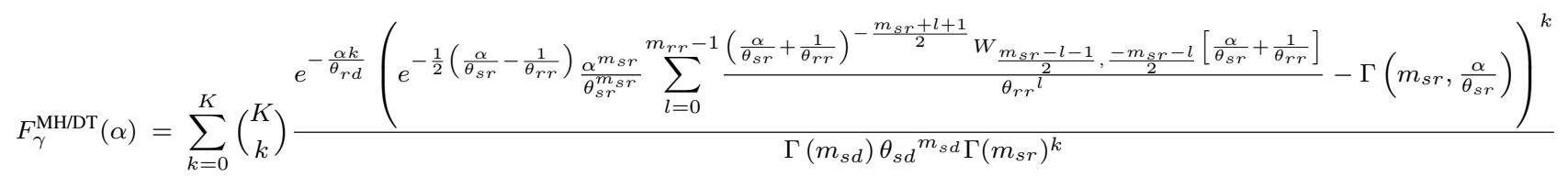

$$
\begin{aligned}
& \times \sum_{\sum_{n=1}^{m_{r d}} k_{n}=k} \frac{\Gamma(k+1)\left(\frac{\alpha}{\theta_{r d}}\right)^{\sum_{n=1}^{m_{r d}} k_{n}(n-1)}}{\prod_{n=1}^{m_{r d}} \Gamma\left(k_{n}+1\right) \Gamma(n)^{k_{n}}} \sum_{r=0}^{\sum_{n=1}^{m_{r d}} k_{n}(n-1)}\left(\begin{array}{c}
\sum_{n=1}^{m_{r d}} k_{n}(n-1) \\
r
\end{array}\right) \eta_{k}^{-\left(r+m_{s d}\right)} \gamma\left(r+m_{s d}, \alpha \eta_{k}\right) .
\end{aligned}
$$

\section{NumericAl Results}

In this section, we numerically evaluate the performance of full-duplex relay selection (FDRS), and verify the theoretical findings derived in previous sections. All numerical results are evaluated by averaging over $10^{7}$ sets of channel realizations with the parameters summarized in the caption of each figure. Also, for clarity of presentation, solid lines with unfilled marks are used to plot the theoretical results, while the same filled marks with no connecting lines are used for simulation results. Hence, curves with solid lines and filled marks indicate perfect matching between theoretical and simulation results.

\section{A. Outage Probability}

In Fig. 2, the outage performance of DF-FDRS is shown for the three schemes under consideration. As expected, when opportunistic relay selection is considered only among the available $K$ dual-hop paths, the performance of the system can be seriously degraded as soon as a direct link starts to get into the picture. This can be seen from the relative performance of the NDL and IDL schemes, which is caused by the introduced direct-link interference in all second hops. On the other hand, when the direct link is also considered as an additional diversity branch, the performance can be significantly enhanced to the extent that the hybrid MH/DT scheme may even outperform the NDL case as shown.

\section{B. Throughput}

In Fig. 3, we plot the throughput versus the fixed transmission rate of the source in bits/sec/Hz. For fixed-rate transmission, the end-to-end throughput, $\mathcal{T}$, is obtained simply as

$$
\mathcal{T}=R\left(1-\mathcal{P}_{\text {out }}\right) \text { bits } / \mathrm{sec} / \mathrm{Hz},
$$

where $R$ is the fixed source transmission rate in bits/sec/Hz, and $\mathcal{P}_{\text {out }}$ is the end-to-end outage probability when the source rate is equal to $R$. The shown figure emphasizes the performance gap between the NDL and IDL scenarios. As shown, exploiting the direct link as an additional non-cooperative path in the hybrid MH/DT scheme offers a significant throughput

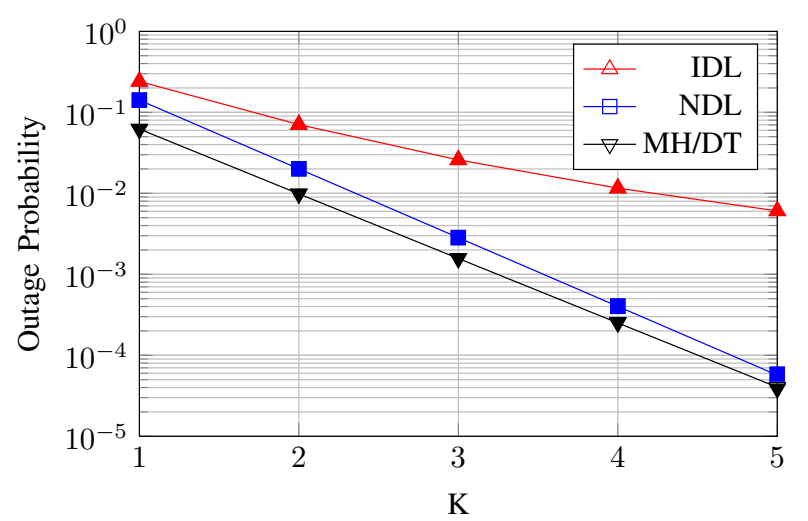

Fig. 2. Outage probability vs. $K$, for $\left(\pi_{s r}, \pi_{r d}, \pi_{r r}, \pi_{s d}\right)=(10,10,3,3)$ $\mathrm{dB},\left(m_{s r}, m_{r d}, m_{r r}, m_{s d}\right)=(2,2,3,1)$ and $R=1 \mathrm{bit} / \mathrm{sec} / \mathrm{Hz}$.

enhancement relative to the IDL scheme even with treating direct link as interference to all other dual-hop paths. This is due to the elimination of the particular error event caused by having large-valued realizations of the direct-link gain which prohibit the communication via all cooperative dual-hop paths while direct transmission can take place.

It has been found that the fading severity of the RSI link, $m_{r r}$, is of minimal effect on the end-to-end performance in the scenarios of practical interest. Specifically, when the average gain of the first hop is well above that of the RSI link, it was found that the outage performance almost does not change. The performance can be slightly affected only in the scenarios where the RSI link gain approaches that of the $\mathrm{S}-\mathrm{R}$ link. However, the end-to-end communication in the latter scenarios tends to fail due to the communication failure in the first hop. This result agrees with a recent result that has been reported in [13] for the single relay scenario.

\section{CONClusion}

In this work, exact expressions for the cumulative distribution function of the end-to-end signal-to-noise-ratio were 


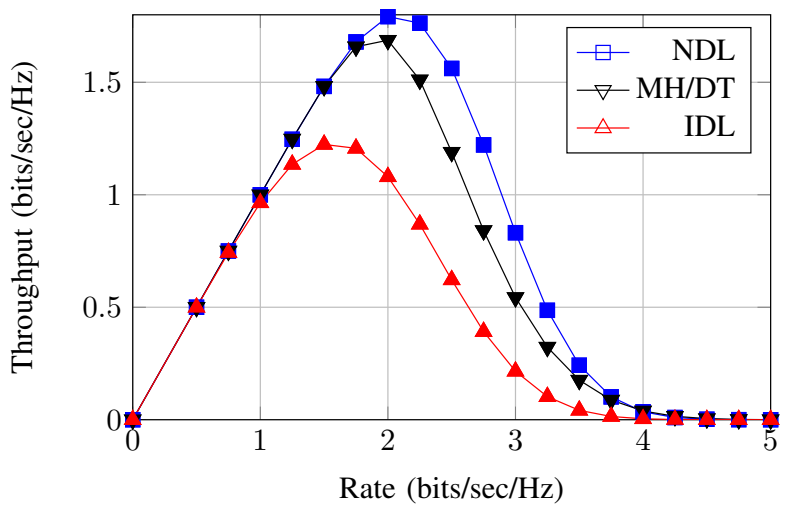

Fig. 3. Throughput vs. rate, for $\left(\pi_{s r}, \pi_{r d}, \pi_{r r}, \pi_{s d}\right)=(10,10,3,5) \mathrm{dB}$, $\left(m_{s r}, m_{r d}, m_{r r}, m_{s d}\right)=(2,2,3,1)$ and $K=4$ relays.

derived for opportunistic decode-and-forward full-duplex relay selection, taking the self-interference of the relays into account. The performance was analyzed when the different links experience general Nakagami- $m$ fading, and compared in two relaying scenarios where either coverage extension or throughput enhancement is targeted. The derived exact analytical results were shown to perfectly match with those obtained via numerical simulations. In the scenarios where a direct source-to-destination link exists, and even when the direct link is treated as interference to all dual-hop paths, it was shown that considering direct transmission as a possible diversity branch has the potential to offer significant performance enhancements that can reach and may exceed the scenario with no direct link.

\section{REFERENCES}

[1] S. Hong, J. Brand, J. Choi, M. Jain, J. Mehlman, S. Katti, and P. Levis, "Applications of self-interference cancellation in 5G and beyond," IEEE Commun. Mag., vol. 52, no. 2, pp. 114-121, Feb. 2014.

[2] M. Jain, J. Choi, T. Kim, D. Bharadia, S. Seth, K. Srinivasan, P. Levis, S. Katti, and P. Sinha, "Practical, real-time, full duplex wireless," in Proc. ACM MobiCom'11, Las Vegas, NV, Sept. 2011.

[3] M. Duarte, C. Dick, and A. Sabharwal, "Experiment-driven characterization of full-duplex wireless systems," IEEE Trans. Wireless Commun., vol. 11, no. 12, pp. 4296-4307, Dec. 2012.
[4] E. Everett, A. Sahai, and A. Sabharwal, "Passive self-interference suppression for full-duplex infrastructure nodes," IEEE Trans. Wireless Commun., vol. 13, no. 2, pp. 680-694, Feb. 2014.

[5] E. Ahmed, A. M. Eltawil, Z. Li, and B. A. Cetiner, "Full-duplex systems using multi-reconfigurable antennas," submitted to IEEE Trans. Wireless Commun., [Online]. Available: http://arxiv.org/abs/1405.7720.

[6] T. Riihonen, S. Werner, and R. Wichman, "Hybrid full-duplex/halfduplex relaying with transmit power adaptation," IEEE Trans. Wireless Commun., vol. 10, no. 9, pp. 3074-3085, Sept. 2011.

[7] I. Krikidis, H.A. Suraweera, P. J. Smith, and C. Yuen, "Full-duplex relay selection for amplify-and-forward cooperative networks," IEEE Trans. Wireless Commun., vol. 11, no. 12, pp. 4381-4393, Dec. 2012.

[8] T. Kwon, S. Lim, S. Choi, and D. Hong, "Optimal duplex mode for DF relay in terms of the outage probability," IEEE Trans. Veh. Technol., vol. 59 , no. 7, pp. $3628-3634$, Sept. 2010.

[9] H. Alves, G. Fraidenraich, R.D. Souza, M. Bennis, and M. Latva-aho, "Performance analysis of full duplex and selective and incremental half duplex relaying schemes," in Proc. IEEE WCNC'12, Apr. 2012.

[10] M. Khafagy, A. Ismail, M.-S. Alouini, and S. Aïssa, "On the outage performance of full-duplex selective decode-and-forward relaying," IEEE Commun. Lett., vol. 17, no. 6, pp. 1180-1183, June 2013.

[11] P. K. Sharma and P. Garg, "Outage analysis of full duplex decode and forward relaying over Nakagami- $m$ channels," in Communications (NCC), 2013 National Conference on, New Delhi, India, Feb. 2013.

[12] H. Alves, D.B. da Costa, R.D. Souza, and M. Latva-aho, "Performance of block-Markov full duplex relaying with self interference in Nakagami-m fading," IEEE Wireless Commun. Lett., vol. 2, no. 3, pp. 311-314, June 2013.

[13] M. G. Khafagy, A. Ismail, M.-S. Alouini, and S. Aïssa, "Efficient cooperative protocols for full-duplex relaying over nakagami- $m$ fading channels," IEEE Trans. Wireless Commun., 2015.

[14] B. Zhong, D. Zhang, Z. Zhang, Z. Pan, K. Long, and A.V. Vasilakos, "Opportunistic full-duplex relay selection for decode-and-forward cooperative networks over rayleigh fading channels," in Proc. IEEE ICC'14, June 2014, pp. 5717-5722.

[15] B. Zhong, Z. Zhang, X. Chai, Z. Pan, K. Long, and H. Cao, "Performance analysis for opportunistic full-duplex relay selection in underlay cognitive networks," IEEE Trans. Veh. Technol., 2014.

[16] M. G. Khafagy, M.-S. Alouini, and S. Aïssa, "Full-duplex opportunistic relay selection in future spectrum-sharing networks," in Proc. IEEE ICC'15 Wkshps, London, UK, June 2015, accepted for publication.

[17] M. Abramowitz and I. A. Stegun, Eds., Handbook of Mathematical Functions, $10^{\text {th }}$ Printing, Dover Publications, Dec. 1972.

[18] J. Lee, H. Wang, J. G. Andrews, and D. Hong, "Outage probability of cognitive relay networks with interference constraints," IEEE Trans. Wireless Commun., vol. 10, no. 2, pp. 390-395, Feb. 2011.

[19] I. S. Gradshteyn and I. M. Ryzhik, Table of Integrals, Series, and Products, Seventh Edition, Academic Press, 2007. 